\title{
Psychological and Pedagogical Foundations for a Computer-Based Learning Environment with a Language-Deficient Domain
}

\author{
Ah Lian Kor \\ School of Computing, University of Leeds, Leeds LS2 9JT, UK \{Lian@Comp.leeds.ac.uk\}
}

\begin{abstract}
In a language-deficient domain such as buoyancy, students generally find it difficult to explain phenomena that daily saturate their lives such as sinking and floating. To address this problem, we propose a simple and object-related articulation and reflection tool which is embedded in the BSL System (B stands for Body while $S$ and $L$ are for String and Liquid respectively). An analysis of the findings reveals that generally, the use of the tool decreased with respect to time. Evidence also shows that contents in the tool is either adapted or misused. Finally, evidence suggests positive changes in students' conceptual knowledge of $B$ and $S$ but not $L$.
\end{abstract}

\section{Introduction}

In language-deficient domains such as buoyancy, students find it difficult to verbalise their thoughts. Thus, we propose an articulation and reflection tool which is phrased in simple object-related language. In this paper, we are going to discuss the psychological and pedagogical bases for the BSL System. Next, the features in the BSL System shall be described. Lastly, the usage and effectiveness of the embedded articulation and reflection tool shall be investigated.

\section{Psychological Foundation}

\subsection{Vygotsky's notion of Scientific versus Spontaneous Concepts}

The development of scientific concepts first begins with verbal definitions, which tends to 'descend' to the concrete that is the phenomena the concept represents [4]. On the contrary, everyday concepts tend to 'ascend' towards abstraction and generalisation. Although spontaneous concepts saturate a learner's experience their definition is extremely difficult to formulate. We call this the 'language deficiency syndrome'.

\section{Pedagogical Foundations}

\subsection{Learning through Articulation}

When cognitive concepts and processes are articulated, they are thereby explicated, and also become an object of reflection which further fosters the generalisation and abstraction processes [2]. This coupling of the articulation and reflection processes is called the 'articulate reflection' and it is suggested that in computer-based learning systems, these two processes should go hand in hand in order to effect better learning [5].

\subsection{Learning through Reflection}

Experience alone is not the key to learning, and reflection is one activity that could transform experience to learning [1][3]. Kolb's experiential learning cycle [3] shows that concrete experience is turned into abstract concepts and generalisations through observations and reflection.

\section{BSL System}

One of its aims is to foster students' better understanding of buoyancy and it consists of three stages: Introduction Stage, Questions Stage, and ProblemSolving Stage.

\subsection{Introduction Stage}

The system first introduces the objects of the laboratory model: Body, String, and Liquid followed by defining their respective forces in simple lay terms. In the Articulation-cum-Reflection Tool, Body Force (B) is defined as 'Weight of the body'; String Force (S), 'Force in the string which prevents the body from falling'; Liquid Force $(L)$, 'Force in the liquid which supports the body'.

\subsection{Questions Stage}

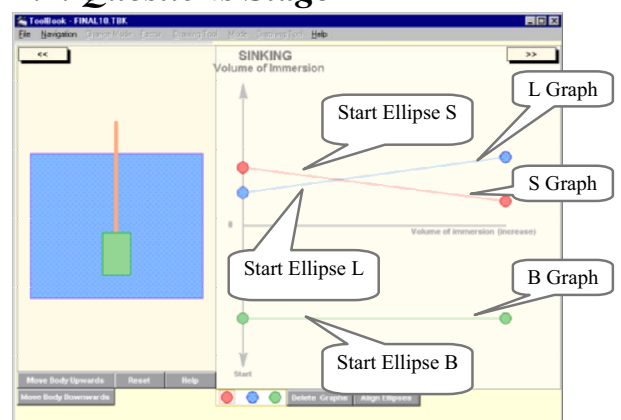

Figure 1. An example of a predicted solution

When students manipulate an attribute of the body or liquid (e.g. density, volume, shape, volume of immersion, etc.), the model changes accordingly. As the student explores the system, he articulates and reflects on the causal effect of the manipulation on BSL. Every prediction of causal relations has to be justified. A twodimensional qualitative graph is employed to represent students' predicted solutions (Figure 1). When students perform the tasks in the Questions Stage, they are actually demonstrating Vygotsky's notion of 'ascend' process of concepts where prior knowledge and spontaneous concepts are harnessed for the prediction of solutions to problems. 
4.3. Problem-Solving Stage

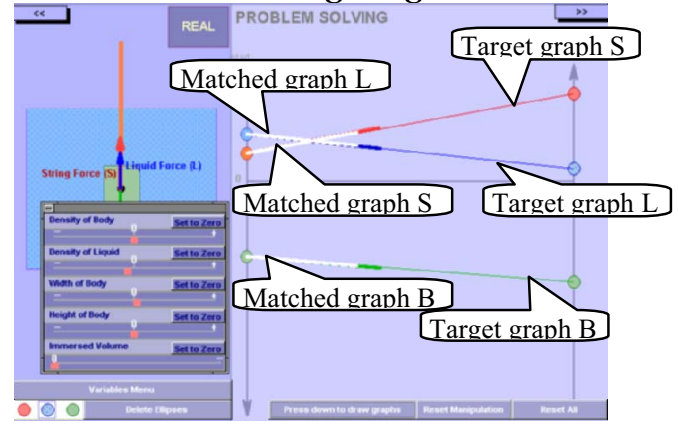

Figure 2. Comparison of target and matched graphs

Tasks in this final stage exemplify the 'descend' process since it involves experimentation and evaluation of hypotheses. Students begin their exploration by creating problems in the form of two-dimensional qualitative target graphs shown in Figure 2. They are requested to predict and justify the solutions for the created problems. Subsequently, one or more of the following variables have to be manipulated: Density of Body, Density of Liquid, Width of Body, Height of Body, and Immersed Volume of Body (Figure 2) in order to obtain a correct solution. Students run the experiment to confirm the correctness of the solution. As the experiment is run, students will be able to observe appropriate changes in the laboratory model, the BSL forces, and concurrently view the situational feedback represented by the matched graphs which are automatically generated (Figure 2). When both the target and matched graphs coincide then the solution is confirmed to be correct.

\section{A Summary of Findings}

\subsection{Articulation}

The research design is a form of observational case study. In the experiment, all the nine participants were final year undergraduate engineering students and their thinking aloud protocols were audio-taped.

Results reveal that the amount of articulation generally decreases with respect to time. Typically, peaks occur at the outset of the exploration of the BSL System due to cognitive load. Sometimes, peaks occur when prior knowledge causes confusion. Evidence also shows that informal language is extensively employed in students' articulation. An analysis of the articulation content reveals that some of the terms in the Articulation-cumReflection Tool have been adapted or misused.

\subsection{Learning}

Evidence suggests that the Articulation-cumReflection Tool effects positive changes in students' conceptual knowledge of $\mathrm{B}$. Generally, S is initially perceived as a dependent variable of B or L. However, as the students progress through a series of tasks, $\mathrm{S}$ is perceived as a dependent variable of both $\mathrm{B}$ and $\mathrm{L}$. Nevertheless, throughout the entire exploration of the system, evidence suggests that students' conceptual knowledge of $\mathrm{L}$ is predominantly devoid of the essential concepts that constitute Archimedes' Principle.

\section{Conclusion}

Evidence has shown that the Articulation-cumReflection Tool has been used by students whilst exploring the system. It also has effected some form of learning. Viewing the potential benefits of similar tools in other language-deficient domains, we propose a list of essential characteristics for such tools. Terms used ought to be simple and object-related so as to invoke relevant spontaneous concepts and definitions should be phrased in lay terms so as to facilitate easy understanding of concepts.

\section{References}

[1] Dewey, J. (1960). How we think: A restatement of the relation of reflective thinking to the education process. Lexington: Heath.

[2] Gruber, H., Law, L. C., Mandl, H., \& Renkl, A. (1995). Situated learning and transfer. In Reimann, P., \& Spada, H. (eds.), Learning in humans and machines. Oxford: Pergamon, pp. 168-188.

[3] Kolb, D. A., \& Fry, R. (1975). Towards an applied theory of experiential learning. In Cooper, C. L. (ed.), Theories of group processes. London: John Wiley, pp. 33-58.

[4] Moll, L. C. (1990). Introduction. In Moll, L. C. (ed.). Vygotsky and education: Instructional implications and applications of sociohistorical psychology. Cambridge: Cambridge University Press, pp. 1-30.

[5] Self, J., Karakirik, E., Kor, A. L., Tedesco, P., \& Dimitrova, V. (2000). Computer-based strategies for articulation reflection (and reflective articulation). Proceedings of ICCE/ICCAI 2000, 21-24 November, 2000, Taipei, Taiwan, pp. 3-12. 